Journal of Innovative Optical Health Sciences

Vol. 9, No. 6 (2016) 1699001 (6 pages)

(c) World Scientific Publishing Company

DOI: $10.1142 / \mathrm{S} 1793545816990017$

\title{
Author Index Volume 9 (2016)
}

Agarwal, R., see Veer, K.

Artemyev, D. N., see Gao, W.

Bagratashvili, V., see Kuznetsova, D.

Bai, J., see Lv, Y.

Balakrishnan, U., Venkatachalapathy, K. and Marimuthu, G. S., An Enhanced PSO-DEFS Based Feature

Selection with Biometric Authentication for Identification of Diabetic Retinopathy

Bityurin, N., see Kuznetsova, D.

Bogatyrev, V. A., see Bucharskaya, A. B.

Bogatyrev, V. A., see Bucharskaya, A. B.

Bratchenko, I. A., see Gao, W.

Bredikhin, V., see Kuznetsova, D.

Bucharskaya, A. B., Maslyakova, G. N., Pakhomy, S. S., Zlobina, O. V., Bugaeva, I. O., Navolokin,

N. A., Khlebtsov, B. N., Bogatyrev, V. A., Khlebtsov, N. G. and Tuchin, V. V., The Morphological

Changes in the Internal Organs of Laboratory Animals After Prolonged Oral Administration

of Gold Nanoparticles

Bucharskaya, A. B., Pakhomy, S. S., Zlobina, O. V., Maslyakova, G. N., Matveeva, O. V.,

Bugaeva, I. O., Navolokin, N. A., Khlebtsov, B. N., Bogatyrev, V. A., Khlebtsov,

N. G. and Tuchin, V. V., Alterations of Morphology of Lymphoid Organs and Peripheral Blood

Indicators Under the Influence of Gold Nanoparticles in Rats

Bugaeva, I. O., see Bucharskaya, A. B.

Bugaeva, I. O., see Bucharskaya, A. B.

Cai, W., see Huang, L.

Cao, L., see Yang, Q.

Cao, X., see Chen, D.

Cao, Y., see Feng, X.

Cao, Y., see $\mathrm{Li}, \mathrm{X}$.

Cao, Y., see Wang, Y.

Cao, Y., see Wei, X.

Carvalho, M. I., see Oliveira, L.

Chaturvedi, P., see Sahu, A.

Chen, C., see Feng, X.

Chen, D., Wang, Y., Li, B., Lin, H., Lin, X. and Gu, Y., Effects of Pulse Width and Repetition Rate of Pulsed Laser on Kinetics and Production of Singlet Oxygen Luminescence

Chen, D., Wu, Y., Sui, T., Zeng, S., Cao, X. and Lv, X., DIC Imaging for Identification of Motor and Sensory Nerves

Chen, G., see Fang, W.

Chen, J., Qiu, C., You, M., Chen, X., Yang, H. and Xie, S., Structured Illumination Microscopy and Its New Developments

Chen, J., see Qiu, C.

Chen, J., Tong, T. and Wang, H., Super-Resolution Imaging in Glycoscience: New Developments and Challenges

Chen, M., see Li, C.

Chen, W. R., see Zhou, F.

Chen, X., see Chen, J.

Chen, X., see Wang, J.

Chen, Z., see He, Q.

9 (2016) 1650025

9 (2016) 1650003

9 (2016) 1650011

9 (2016) 1650010

9 (2016) 1650020

9 (2016) 1650011

9 (2016) 1642004

9 (2016) 1640004

9 (2016) 1650003

9 (2016) 1650011

9 (2016) 1642004

9 (2016) 1640004

9 (2016) 1640004

9 (2016) 1642004

9 (2016) 1650015

9 (2016) 1630002

9 (2016) 1643001

9 (2016) 1550039

9 (2016) 1650009

9 (2016) 1650022

9 (2016) 1650016

9 (2016) 1650035

9 (2016) 1650017

9 (2016) 1550039

9 (2016) 1650019

9 (2016) 1643001

9 (2016) 1650037

9 (2016) 1630010

9 (2016) 1641001

9 (2016) 1630007

9 (2016) 1650001

9 (2016) 1630001

9 (2016) 1630010

9 (2016) 1630003

9 (2016) 1650006 
Chen, Z., see Liang, L.

9 (2016) 1650018

Cheng, Z., see Zhao, N.

Cunha, Â., see Gomes, M. C.

9 (2016) 1630004

9 (2016) 1630005

9 (2016) 1650017

Deshmukh, A., see Sahu, A.

9 (2016) 1642003

$\mathrm{Du}, \mathrm{B}$., see Jiang, $\mathrm{X}$.

Duan, L., see Fang, W.

Elagin, V., see Kuznetsova, D.

Fang, W. and Wei, Y., Upconversion Nanoparticle as a Theranostic Agent for Tumor Imaging and Therapy

9 (2016) 1650037

9 (2016) 1650011

9 (2016) 1630006

Fang, W., Feng, H., Yang, W., Duan, L., Chen, G., Xiong, L. and Liu, Q., High-Throughput

9 (2016) 1650037

Volumetric Reconstruction for 3D Wheat Plant Architecture Studies

Feng, H., see Fang, W.

Feng, X., Cao, Y., Peng, K. and Chen, C., An Online Identity Authentication Method for Blood Smear

9 (2016) 1650037

9 (2016) 1550039

9 (2016) 1630009

9 (2016) 1650015

Gao, F., see Huang, L.

Gao, W., Zakharov, V. P., Myakinin, O. O., Bratchenko, I. A., Artemyev, D. N. and Kornilin, D. V., Medical Images Classification for Skin Cancer Using Quantitative Image Features with Optical Coherence Tomography

Gao, X., see Zhang, Y.

Gnyba, M., see Karpienko, K.

Golnaraghi, F., see Shokoufi, M.

Gomes, M. C., Cunha, Â., Trindade, T. and Tomé, J. P. C., The Role of Surface Functionalization of Silica Nanoparticles for Bioimaging

$\mathrm{Gu}, \mathrm{Y} .$, see Chen, D.

Guang, H., see Lv, Y.

Gun'kov, V. V., see Maryakhina, V. S.

Guo, H., see He, X.

Guo, Z., see Xiong, H.

He, Q., Liang, L., Chen, Z., Pang, Q. and Zhao, J., Study of the Best Decocting Time of Sun

Dried Ginseng by Using the Hyperspectral Imaging Technology

He, Q., see Liang, L.

He, X., Guo, H., Yu, J., Zhang, X. and Hou, Y., Effective and Robust Approach for Fluorescence Molecular Tomography Based on CoSaMP and $\mathrm{SP}_{3}$ Model

He, Y., see Sun, Y.

Hole, A. R., see Sahu, A.

Hong, X., Nagarajan, V. K., Mugler, D. H. and Yu, B., Smartphone Microendoscopy for High Resolution Fluorescence Imaging

Hou, Y., see He, X.

Hou, Z., see Qiu, C.

$\mathrm{Hu}, \mathrm{J}$. , see $\mathrm{Xu}, \mathrm{J}$.

Huang, L., Cai, W., Zhao, Y., Wu, D., Wang, L., Wang, Y., Lai, D., Rong, J., Gao, F. and Jiang, H., In Vivo Tumor Detection with Combined MR-Photoacoustic-Thermoacoustic Imaging

Huang, Z., see $\mathrm{Wu}, \mathrm{S}$.

Huang, Z., see Zhang, L.

Huang, Z.-L., see Li, L.

Jędrzejewska-Szczerska, M., see Karpienko, K.

Ji, Y., see Xiong, $\mathrm{H}$.

Jia, X., see Jiang, X.

Jiang, H., see Huang, L.

Jiang, L., see Xu, J.

Jiang, P., see Li, C.

Jiang, X., Du, B., Yu, M., Jia, X. and Zheng, J., Surface-Ligand Effect on Radiosensitization of Ultrasmall Luminescent Gold Nanoparticles

Jin, G., see Yang, Q.

Jin, H., see Lin, Q.

Jin, H., see Sha, S.

Kamensky, V., see Kuznetsova, D.

Kamensky, V. A., see Kazakov, V. V.

Karabut, M., see Kuznetsova, D.

Karpienko, K., Gnyba, M., Milewska, D., Wróbel, M. S. and Jędrzejewska-Szczerska, M., Blood

Equivalent Phantom vs Whole Human Blood, a Comparative Study

9 (2016) 1642005

9 (2016) 1650012

9 (2016) 1650007

9 (2016) 1630005

9 (2016) 1650019

9 (2016) 1650010

9 (2016) 1650005

9 (2016) 1650024

9 (2016) 1650026

9 (2016) 1650006

9 (2016) 1650018

9 (2016) 1650024

9 (2016) 1650008

9 (2016) 1650017

9 (2016) 1650046

9 (2016) 1650024

9 (2016) 1641001

9 (2016) 1641002

9 (2016) 1650015

9 (2016) 1650014

9 (2016) 1640002

9 (2016) 1630008

9 (2016) 1650012

9 (2016) 1650026

9 (2016) 1642003

9 (2016) 1650015

9 (2016) 1641002

9 (2016) 1650001

9 (2016) 1642003

9 (2016) 1630002

9 (2016) 1640001

9 (2016) 1642002

9 (2016) 1650011

9 (2016) 1650013

9 (2016) 1650011

9 (2016) 1650012 
Kazakov, V. V., Sanin, A. G. and Kamensky, V. A., On the Possible Ultrasonic Inspection

9 (2016) 1650013 of Micro-Bubbles Generated by the Optical Fiber Tip

Khlebtsov, B., Prilepskii, A., Lomova, M. and Khlebtsov, N., Au-Nanocluster-Loaded Human

9 (2016) 1650004

Serum Albumin Nanoparticles with Enhanced Cellular Uptake for Fluorescent Imaging

Khlebtsov, B. N., see Bucharskaya, A. B.

Khlebtsov, B. N., see Bucharskaya, A. B.

Khlebtsov, N., see Khlebtsov, B.

Khlebtsov, N. G., see Bucharskaya, A. B.

Khlebtsov, N. G., see Bucharskaya, A. B.

Kittisuwan, P., Image Enhancement via MMSE Estimation of Gaussian Scale Mixture with Maxwell Density in $A W G N$

Kornilin, D. V., see Gao, W.

Krishna, C. M., see Sahu, A.

Kuang, C., see Zhao, G.

Kumar, A., see Veer, K.

Kuznetsova, D., Elagin, V., Karabut, M., Shakhova, M., Bredikhin, V., Snopova, L., Shakhov, A., Sapogova, N., Bityurin, N., Bagratashvili, V. and Kamensky, V., The Influence on Biotissue Laser Resection of a Strongly Absorbing Layer at the Optical Fiber Tip

Lai, D., see Huang, L.

Li, B., see Chen, D.

Li, C., Pastila, R. K. and Lin, C. P., Label-Free Imaging Immune Cells and Collagen in Atherosclerosis with Two-Photon and Second Harmonic Generation Microscopy

Li, C., see Zhang, Y.

Li, C., Shi, X., Chen, M., Xu, G., Su, X., Jiang, P. and Pan, L., Ultraviolet Light A Irradiation Induces Immunosuppression Associated with the Generation of Reactive Oxygen Species in Human Neutrophils

Li, D., see Zhang, Y.

$\mathrm{Li}, \mathrm{H}$., see $\mathrm{Wu}, \mathrm{S}$.

Li, L., Li, M., Zhang, Z. and Huang, Z.-L., Assessing Low-Light Cameras with Photon Transfer Curve Method

Li, M., see Li, L.

$\mathrm{Li}, \mathrm{P}$., see Wang, J.

Li, X. and Cao, Y., A Robust Automatic Leukocyte Recognition Method Based on Island-Clustering Texture Liang, L., see He, Q.

Liang, L., Sun, J., He, Q., Chen, Z., Zhu, S. and Lin, L., Semi-Quantitative Analysis on the Content of Berberine Hydrochloride in Compound Berberine Tablets with the Fluorescence Spectral Imaging Method

Lin, C. P., see Li, C.

Lin, H., see Chen, D.

Lin, L., see Liang, L.

Lin, Q., see Sha, S.

Lin, Q., Sha, S., Yang, F., Jin, H. and Zhang, Z., KillerRed Protein Based In Vivo Photodynamic Therapy and Corresponding Tumor Metabolic Imaging

Lin, X., see Chen, D.

Liu, F., see Lv, Y.

Liu, H., see Zhao, N.

Liu, L., see $\mathrm{Xu}, \mathrm{J}$.

Liu, Q., see Fang, W.

Liu, X., see Zhao, G.

Liu, Y., Lu, X.-M., Niu, Y.-F., Tao, K.-X. and Wang, G.-B., Application of Laparoscopic Total Mesorectal Excision Combined with Sphincter-Preserving Surgery in Low or Ultralow Rectal Cancer

Lomova, M., see Khlebtsov, B.

Lu, X.-M., see Liu, Y.

Luo, J., see Lv, Y.

Lv, X., see Chen, D.

Lv, Y., Zhang, J., Liu, F., Shi, J., Guang, H., Bai, J. and Luo, J., Spectral Selective Fluorescence

Molecular Imaging with Volume Holographic Imaging System

Marimuthu, G. S., see Balakrishnan, U.

Maryakhina, V. S. and Gun'kov, V. V., Change of Photosensitizer Fluorescence at Its Diffusion in Viscous Liquid Flow

Maslyakova, G. N., see Bucharskaya, A. B.

Maslyakova, G. N., see Bucharskaya, A. B.

9 (2016) 1640004

9 (2016) 1642004

9 (2016) 1650004

9 (2016) 1642004

9 (2016) 1640004

9 (2016) 1650021

9 (2016) 1650003

9 (2016) 1650017

9 (2016) 1641003

9 (2016) 1650023

9 (2016) 1650011

9 (2016) 1650015

9 (2016) 1650019

9 (2016) 1640003

9 (2016) 1642005

9 (2016) 1650001

9 (2016) 1642005

9 (2016) 1650014

9 (2016) 1630008

9 (2016) 1630008

9 (2016) 1630003

9 (2016) 1650009

9 (2016) 1650006

9 (2016) 1650018

9 (2016) 1640003

9 (2016) 1650019

9 (2016) 1650018

9 (2016) 1642002

9 (2016) 1640001

9 (2016) 1650019

9 (2016) 1650010

9 (2016) 1630004

9 (2016) 1641002

9 (2016) 1650037

9 (2016) 1641003

9 (2016) 1643002

9 (2016) 1650004

9 (2016) 1643002

9 (2016) 1650010

9 (2016) 1643001

9 (2016) 1650010

9 (2016) 1650020

9 (2016) 1650005

9 (2016) 1640004

9 (2016) 1642004 
Matveeva, O. V., see Bucharskaya, A. B.

9 (2016) 1640004

Milewska, D., see Karpienko, K.

Mugler, D. H., see Hong, X.

Myakinin, O. O., see Gao, W.

Nagarajan, V. K., see Hong, X.

Navolokin, N. A., see Bucharskaya, A. B.

Navolokin, N. A., see Bucharskaya, A. B.

Niu, Y.-F., see Liu, Y.

Nogueira, E., see Oliveira, L.

Nordquist, R. E., see Zhou, F.

Oliveira, L., Carvalho, M. I., Nogueira, E. and Tuchin, V. V., Optical Clearing Mechanisms Characterization in Muscle

Pakhomy, S. S., see Bucharskaya, A. B.

Pakhomy, S. S., see Bucharskaya, A. B.

Pan, L., see Li, C.

Pan, Y., see Zhao, N.

Pang, Q., see He, Q.

Pastila, R. K., see Li, C.

Peng, K., see Feng, X.

Prilepskii, A., see Khlebtsov, B.

Qiao, Y., see Wang, Y.

Qiao, Y., see $\mathrm{Xu}, \mathrm{M}$.

Qin, H., see Yan, B.

Qiu, C., Chen, J., Hou, Z., Xu, C., Xie, S. and Yang, H., Effect of Light Polariztion on Pattern

Illumination Super-Resolution Imaging

Qiu, C., see Chen, J.

Rong, J., see Huang, L.

Rong, Z., see Zhao, G.

Sahu, A., Deshmukh, A., Hole, A. R., Chaturvedi, P. and Krishna, C. M., In Vivo Subsite Classification and Diagnosis of Oral Cancers Using Raman Spectroscopy

Sanin, A. G., see Kazakov, V. V.

Sapogova, N., see Kuznetsova, D.

Sha, S., see Lin, Q.

Sha, S., Yang, F., Wang, A., Jin, H., Zhang, Z. and Lin, Q., Fluorescent and Quantitative

Mitochondrial Redox Imaging of Tumor Targeted by Octa-RGD Probe

Shakhov, A., see Kuznetsova, D.

Shakhova, M., see Kuznetsova, D.

Sharma, T., see Veer, K.

Sharma, T., see Veer, K.

Shi, J., see Lv, Y.

Shi, L., see Zhang, L.

Shi, X., see $\mathrm{Li}, \mathrm{C}$.

Shi, X., see Wang, Y.

Shi, X., see $\mathrm{Xu}, \mathrm{M}$.

Shokoufi, M. and Golnaraghi, F., Development of a Handheld Diffuse Optical Breast Cancer Assessment Probe

Snopova, L., see Kuznetsova, D.

$\mathrm{Su}, \mathrm{X}$., see $\mathrm{Li}, \mathrm{C}$.

Sui, T., see Chen, D.

Sun, J., see Liang, L.

Sun, Y., Zhang, T., Zhao, Y. and He, Y., 3D Automatic Segmentation Method for Retinal Optical

Coherence Tomography Volume Data Using Boundary Surface Enhancement

Tao, K.-X., see Liu, Y.

Tomé, J. P. C., see Gomes, M. C.

Tong, T., see Chen, J.

Trindade, T., see Gomes, M. C.

Tuchin, V. V., see Bucharskaya, A. B.

Tuchin, V. V., see Bucharskaya, A. B.

Tuchin, V. V., see Oliveira, L.

9 (2016) 1650012

9 (2016) 1650046

9 (2016) 1650003

9 (2016) 1650046

9 (2016) 1642004

9 (2016) 1640004

9 (2016) 1643002

9 (2016) 1650035

9 (2016) 1630001

9 (2016) 1650035

9 (2016) 1640004

9 (2016) 1642004

9 (2016) 1650001

9 (2016) 1630004

9 (2016) 1650006

9 (2016) 1640003

9 (2016) 1550039

9 (2016) 1650004

9 (2016) 1550016

9 (2016) 1650002

9 (2016) 1642001

9 (2016) 1641001

9 (2016) 1630010

9 (2016) 1650015

9 (2016) 1641003

9 (2016) 1650017

9 (2016) 1650013

9 (2016) 1650011

9 (2016) 1640001

9 (2016) 1642002

9 (2016) 1650011

9 (2016) 1650011

9 (2016) 1650023

9 (2016) 1650025

9 (2016) 1650010

9 (2016) 1640002

9 (2016) 1650001

9 (2016) 1550016

9 (2016) 1650002

9 (2016) 1650007

9 (2016) 1650011

9 (2016) 1650001

9 (2016) 1643001

9 (2016) 1650018

9 (2016) 1650008

9 (2016) 1643002

9 (2016) 1630005

9 (2016) 1630007

9 (2016) 1630005

9 (2016) 1642004

9 (2016) 1640004

9 (2016) 1650035 
Veer, K., Sharma, T. and Agarwal, R., A Neural Network-Based Electromyography Motion Classifier

9 (2016) 1650025 for Upper Limb Activities

Veer, K., Sharma, T. and Kumar, A., Development of Prosthetic Arm Using Body Actioned SEMG Signals

Venkatachalapathy, K., see Balakrishnan, U.

Wang, A., see Sha, S.

Wang, G.-B., see Liu, Y.

Wang, H., see Chen, J.

Wang, H., see Xu, J.

Wang, H., see Zhang, L.

Wang, J., Li, P., Chen, X. and Wu, M. X., Laser Facilitates Vaccination

Wang, L., see Huang, L.

Wang, P., see Zhang, L.

Wang, X., see Zhang, L.

Wang, Y. and Cao, Y., A Leukocyte Image Fast Scanning Based on Max-Min Distance Clustering

Wang, Y., see Chen, D.

Wang, Y., see Huang, L.

Wang, Y., see Wu, S.

Wang, Y., Wu, Z., Wang, Z., Xu, M., Shi, X. and Qiao, Y., Rapid Analysis of Spatial Distribution of PVPP and Hardness of Yinhuang Dispersible Tablets by NIR-CI

Wang, Z., see Wang, Y.

Wei, X. and Cao, Y., Automatic Counting Method for Complex Overlapping Erythrocytes Based on Seed Prediction in Microscopic Imaging

Wei, Y., see Fang, W.

Wróbel, M. S., see Karpienko, K.

$\mathrm{Wu}, \mathrm{D}$., see Huang, L.

Wu, M. X., see Wang, J.

Wu, S., Huang, Z., Wang, Y. and Li, H., Characterizing UVB-Induced Skin Tumor Process Using Optical Coherence Tomography

$\mathrm{Wu}, \mathrm{Y}$. , see Chen, D.

Wu, Z., see Wang, Y.

$\mathrm{Wu}, \mathrm{Z}$. , see $\mathrm{Xu}, \mathrm{M}$.

Xie, S., see Chen, J.

Xie, S., see Qiu, C.

Xiong, H., Guo, Z., Zhong, H. and Ji, Y., Monitoring the Penetration and Accumulation of Gold

Nanoparticles in Rat Skin Ex Vivo Using Surface-Enhanced Raman Scattering Spectroscopy

Xiong, L., see Fang, W.

$\mathrm{Xu}, \mathrm{C}$, see Qiu, C.

$\mathrm{Xu}, \mathrm{G}$., see $\mathrm{Li}, \mathrm{C}$.

Xu, J., Jiang, L., Zhu, H., Liu, L., Hu, J., Wang, H. and Zhuang, S., Experimental Generation and Observation of a Super-Resolution Optical Tube

$\mathrm{Xu}$, M., see Wang, Y.

Xu, M., Zhou, L., Zhang, Q., Wu, Z., Shi, X. and Qiao, Y., Near-Infrared Chemical Imaging for Quantitative Analysis of Chlorpheniramine Maleate and Distribution Homogeneity Assessment in Pharmaceutical Formulations

$\mathrm{Xu}, \mathrm{P}$., see Zhang, M.

Yan, B. and Qin, H., Indocyanine Green Loaded Graphene Oxide for High-Efficient Photoacoustic Tumor Therapy

Yang, F., see Lin, Q.

Yang, F., see Sha, S.

Yang, H., see Chen, J.

Yang, H., see Qiu, C.

Yang, Q., Cao, L., Zhang, H., Zhang, H. and Jin, G., Method of Lateral Image Reconstruction in Structured Illumination Microscopy with Super Resolution

Yang, W., see Fang, W.

You, M., see Chen, J.

$\mathrm{Yu}, \mathrm{B}$., see Hong, X.

$\mathrm{Yu}, \mathrm{J} .$, see $\mathrm{He}, \mathrm{X}$.

$\mathrm{Yu}, \mathrm{M}$., see Jiang, X.

Zakharov, V. P., see Gao, W.

Zeng, S., see Chen, D.

9 (2016) 1650023

9 (2016) 1650020

9 (2016) 1642002

9 (2016) 1643002

9 (2016) 1630007

9 (2016) 1641002

9 (2016) 1640002

9 (2016) 1630003

9 (2016) 1650015

9 (2016) 1640002

9 (2016) 1640002

9 (2016) 1650022

9 (2016) 1650019

9 (2016) 1650015

9 (2016) 1650014

9 (2016) 1550016

9 (2016) 1550016

9 (2016) 1650016

9 (2016) 1630006

9 (2016) 1650012

9 (2016) 1650015

9 (2016) 1630003

9 (2016) 1650014

9 (2016) 1643001

9 (2016) 1550016

9 (2016) 1650002

9 (2016) 1630010

9 (2016) 1641001

9 (2016) 1650026

9 (2016) 1650037

9 (2016) 1641001

9 (2016) 1650001

9 (2016) 1641002

9 (2016) 1550016

9 (2016) 1650002

9 (2016) 1630009

9 (2016) 1642001

9 (2016) 1640001

9 (2016) 1642002

9 (2016) 1630010

9 (2016) 1641001

9 (2016) 1630002

9 (2016) 1650037

9 (2016) 1630010

9 (2016) 1650046

9 (2016) 1650024

9 (2016) 1642003

9 (2016) 1650003

9 (2016) 1643001 
Zhang, G., see Zhang, L.

Zhang, H., see Yang, Q.

Zhang, H., see Yang, Q.

Zhang, J., see Lv, Y.

Zhang, L., Wang, P., Shi, L., Zhang, G., Zhang, Y., Zhou, Z., Huang, Z., Wang, H. and Wang, X., Topical 5-Aminolevulinic Acid Photodynamic Therapy Improved Refractory acne Conglobata and Perifolliculitis Capitis Abscedens et Suffodiens Rather than Hidradenitis Suppurativa

Zhang, M., Fu, Z. and Xu, P., Extending the Spatiotemporal Resolution of Super-Resolution

Microscopies Using Photomodulatable Fluorescent Proteins

Zhang, Q., see Xu, M.

Zhang, T., see Sun, Y.

Zhang, X., see He, X.

Zhang, Y., Li, D., Zhou, X., Gao, X., Zhao, S. and Li, C., Enhancing Sensitivity of SERRS Nanoprobes by Modifying Heptamethine Cyanine-Based Reporter Molecules

Zhang, Y., see Zhang, L.

Zhang, Z., see Li, L.

Zhang, Z., see Lin, Q.

Zhang, Z., see Sha, S.

Zhao, G., Rong, Z., Kuang, C., Zheng, C. and Liu, X., 3D Fluorescence Emission Difference

Microscopy Based on Spatial Light Modulator

Zhao, J., see He, Q.

Zhao, N., Pan, Y., Cheng, Z. and Liu, H., Gold Nanoparticles for Cancer Theranostics: A Brief Update

Zhao, S., see Zhang, Y.

Zhao, Y., see Huang, L.

Zhao, Y., see Sun, Y.

Zheng, C., see Zhao, G.

Zheng, J., see Jiang, X.

Zhong, H., see Xiong, H.

Zhou, F., Nordquist, R. E. and Chen, W. R., Photonics Immunotherapy - A Novel Strategy for Cancer Treatment

Zhou, L., see $\mathrm{Xu}, \mathrm{M}$.

Zhou, X., see Zhang, Y.

Zhou, Z., see Zhang, L.

Zhu, H., see $\mathrm{Xu}$, J.

Zhu, S., see Liang, L.

Zhuang, S., see $\mathrm{Xu}$, J.

Zlobina, O. V., see Bucharskaya, A. B.

Zlobina, O. V., see Bucharskaya, A. B.
9 (2016) 1640002

9 (2016) 1630002

9 (2016) 1630002

9 (2016) 1650010

9 (2016) 1640002

9 (2016) 1630009

9 (2016) 1650002

9 (2016) 1650008

9 (2016) 1650024

9 (2016) 1642005

9 (2016) 1640002

9 (2016) 1630008

9 (2016) 1640001

9 (2016) 1642002

9 (2016) 1641003

9 (2016) 1650006

9 (2016) 1630004

9 (2016) 1642005

9 (2016) 1650015

9 (2016) 1650008

9 (2016) 1641003

9 (2016) 1642003

9 (2016) 1650026

9 (2016) 1630001

9 (2016) 1650002

9 (2016) 1642005

9 (2016) 1640002

9 (2016) 1641002

9 (2016) 1650018

9 (2016) 1641002

9 (2016) 1640004

9 (2016) 1642004 